\title{
Metodología de medición de los aprovechamientos urbanísticos en función del modelo de desarrollo urbano. Caso de estudio: Sogamoso, Colombia*
}

\author{
Measuring urban use in Sogamoso, Colombia: \\ a methodological proposal
}

Tadeo Humberto Sanabria Artunduaga ${ }^{* * *}$ John Fredy Ramírez Ríos****

Álvaro Javier Bolaños Palacios ${ }^{* * * * * * *}$

Citar este artículo como: Sanabria, T. H.; Ramírez, J. F.; Bolaños, A. J. (2016) "Metodología de medición de los aprovechamientos urbanísticos en función del modelo de desarrollo urbano". Revista Nodo, 10(20), pp. 38-54.

\section{Resumen}

Se propone una metodología de medición del Aprovechamiento Urbanístico (AU) en función del modelo de desarrollo urbano. Se toma como caso de estudio el Plan de Ordenamiento Territorial (POT) de la ciudad de Sogamoso (Boyacá, Colombia) que fue formulado en el año 2000, y su correspondiente Código Urbano (CU) decretado en el año 2002. En dicho POT se formuló un Modelo de Desarrollo Urbano (MDU) basado en principios discursivos relacionados con sostenibilidad, competitividad y equidad, a los cuales se les asignaron dimensiones cuantitativas que permitieran su medición en los aprovechamientos urbanísticos (AU) permitidos por el CU. Luego se procedió a simular lo que dicho POT diagnosticó de la realidad urbana de tres áreas previamente delimitadas; $y$ una segunda que proyecta en dichas áreas los máximos AU permitidos por el (CU), y llevar a cabo una comparación cuantitativa de ambas simulaciones. El procedimiento arroja unos resultados que permiten evaluar de manera cuantitativa la correspondencia entre la materialización física de un aprovechamiento y el discurso que teóricamente lo soporta.

\section{Palabras clave}

Plan de Ordenamiento Territorial, Código Urbano, Desarrollo Urbano, Modelo Urbano, Aprovechamientos Urbanísticos.

Fecha de recepción: 31 de marzo de 2016 Fecha de aceptación: Mayo 26 de 2016

* Artículo de investigación científica y tecnológica.

\#: Arquitecto. Magíster en Gestión y Valoración Urbana. Magíster en Ordenamiento Urbano y Regional. Docente Investigador. Fundación Universidad de América. Grupo de Investigación Prospectiva Urbano-Territorial. Correo: Tadeo.sanabria@investigadores.uamerica.edu.co.

: Sociólogo. Magíster en Urbanismo. Docente Investigador. Fundación Universidad de América. Grupo de Investigación Prospectiva Urbano-Territorial. Correo: john.ramirez@investigadores.uamerica.edu.co

***** Arquitecto. Magíster en Urbanismo. Docente Investigador. Fundación Universidad de América. Grupo de Investigación Prospectiva Urbano-Territorial. Correo: alvaro.bolanos@investigadores.uamerica.edu.co 


\section{Abstract}

A spatial analysis of quantitative interactions between theory and practice in urban planning is proposed. Case Study: Sogamoso's Spatial Plan (called in Colombia Plan de Ordenamiento Territorial) and its urban code (UC). An urban model of development based on discursive principles related to territorial sustainability, regional competitiveness and spatial equity is described. Measurable dimensions are applied upon those principles by using two 3D comparable simulations. The first simulation reproduces the current reality in three zones previously chosen. The second one creates new city forms in those zones by applying the building and construction norms derived from the UC. The comparison of measuring value evidences the emphasis on competitiveness and sustainability in detriment of special equality when urban planning is put into practice.

\section{Keywords}

Spatial planning, urban code, urban development, urban model, urban uses.

\section{Introducción}

La Ley 388 de 1997, Ley de Desarrollo Territorial (LDT), obliga a los municipios colombianos a formular un Plan de Ordenamiento Territorial (POT). En éste se formula un Modelo Territorial de Desarrollo Urbano (MDU), que suele invocar discursos de todo tipo, entre los que se destacan el fomento de la competitividad municipal y regional, la sostenibilidad ambiental, la equidad, y la justicia social $^{1}$. Por otra parte, debe acompañar a la formulación del POT, un código de urbanismo (CU), que regule física y espacialmente los Aprovecha-

1 Los cuales usualmente son sugeridos e incorporados por los consultores que son contratados para liderar los procesos de formulación de los POT, y que son conocedores (en diversos grados) de las tendencias mundiales en planeación y desarrollo urbano-regional. mientos Urbanísticos (AU). Un aprovechamiento de acuerdo con el numeral 1 del Artículo 2 del Decreto 2181 es "el número de metros cuadrados de edificación para un determinado uso que la norma urbanística autoriza en un predio" (Presidencia de la República, 2006).

En ese orden de ideas, se podría suponer que la función de un CU es la de materializar, por medio de una norma edificatoria física o AU, los lineamientos discursivos generales del MDU contenido en un POT. Sin embargo, es difícil establecer cuáles son los parámetros metodológicos que permiten evaluar dicha materialización. Al respecto la Ley simplemente señala que se debe hacer seguimiento al POT por medio de la conformación de un expediente municipal y de sus indicadores, pero no hace referencia como tal a la medición de los AU autorizados. También se han hecho evaluaciones a la Ley 388 y a los instrumentos de planificación que de ella se derivan (Maldonado, 2000; Maldonado, 2008; Molina, 2008), como el mismo POT o los Planes Parciales (PP), pero no existe hasta el momento metodología alguna que aborde la medición cuantitativa y espacializada del AU en función del MDU.

Así pues, en este artículo se propone, usando un caso de estudio, una metodología que mida de manera espacial hasta qué punto los $\mathrm{AU}$ de un $\mathrm{CU}$ decretado en 2002 para la ciudad de Sogamoso (Boyacá, Colombia), consolidan, distorsionan o anulan los principios del MDU propuesto por el POT, y que fue formulado para esa ciudad en el año 2000. El objetivo es ofrecer, por un lado, nuevos insumos al clásico debate de la correspondencia entre la teoría y práctica de la Planeación Urbana-Regional (PUR); y por otro lado, aportar herramientas de evaluación de los Códigos Urbanos, que permitan que las materializaciones constructivas derivadas de las políticas del ordenamiento territorial, correspondan de mejor forma con sus principios discursivos. 


\section{La primacía tradicional de los Códigos Urbanos en Colombia y el surgimiento del POT}

Antes de entrar en detalles sobre la propuesta de metodología de medición de los AU a partir del caso de estudio, es necesario entender de qué manera en Colombia la figura de los CU surgió y se relaciona actualmente con el instrumento POT. Al respecto, se tiene como antecedente que, a comienzos de la década de los cincuenta, Le Corbusier, Wiener y Sert propusieron la creación de unas oficinas del Plan Regulador en los principales centros poblados del país; a partir de las cuales se generaron todo tipo de normativas urbanísticas, entre ellas la del "régimen de alturas y normas para la edificación” (Tarchópulos, 2006). Esas normativas debían ajustarse, en teoría, a las directrices generales de zonificación ofrecidas por un Plan Piloto o Director.

Se inauguró así una tradición de la PUR en Colombia que diferenciaba un nivel general de planificación, en el cual se definía "el modelo de ciudad y las cuestiones relacionadas con la estructura urbana, el centro cívico, tipologías de vivienda, al igual que la zonificación"; y un nivel operativo en el cual se establecía "la forma de la edificación y de los sistemas estructurantes, así como las herramientas de gestión" (Tarchópulos, 2006). Con el paso del tiempo el nivel operativo representado, en parte, por las normas de construcción, se mantendría vigente bajo la figura de los famosos CU, los cuales perderían eventualmente su articulación con niveles generales de planeamiento. Dada esa desarticulación, éstos perdieron su soporte en lineamientos robustos para orientar el desarrollo urbano, excepto la débil pretensión de vincularlos con postulados básicos de zonificación funcional modernista, pero a escala predio a predio (Vejarano, 2012).

A finales de los años setenta, los CU terminarían posicionándose como la única figura efectiva de PUR; desvinculados totalmente de los grandes objetivos de desarrollo urbano propuestos en los planes pilotos y comprehensivos (integrales). Éstos se siguieron formulando durante la mayor parte de la segunda mitad del siglo XX, pero tendrían poca incidencia en su desarrollo físico de los principales centros urbanos del país. Lo que estaría en consonancia con la sentencia de la especialista María C. Vejarano (2012, p. 2) para el caso de Bogotá cuando afirma que "las normas de zonificación han permanecido y han prevalecido, detrás de una variedad amplia de discursos".

A pesar de esa legitimidad tácita y de conveniencia política y económica que se granjearon los $\mathrm{CU}$ a escala municipal, se mostrarían muy insuficientes para ordenar el crecimiento caótico de las ciudades del país a finales de la década de los ochenta. Como ya se dijo, los CU carecían de lineamientos estructurales de urbanismo y de instrumentos de gestión urbana que le dieran la coherencia necesaria a la práctica planificadora. Dada esa situación, a finales de la década de los ochenta, y luego de un largo proceso de concientización social y política "en un medio social y político ultra-conservador que no estaba dispuesto a dejar sus privilegios absolutos sobre la propiedad del suelo urbano y rural" (Ramírez, 2011, p. 94); se abriría paso la primera Ley 9a de Reforma Urbana (1989) impulsada por el entonces senador liberal Ernesto Samper Pizano. Sobre la importancia de esta Ley se afirma lo siguiente:

La experiencia con la Ley 9a contribuyó de manera significativa porque con ella se introdujo una nueva concepción sobre la planificación urbana. Contribuyó a superar la noción de que planificar ciudades era simplemente hacer diseño urbanístico, zonificar usos del suelo y establecer un estatuto procedimental cuyo cumplimiento garantizaba obtener una licencia de construcción; pero no fue suficiente porque no se diseñaron instrumentos eficaces de actuación y aplicación. Precisamente con la Ley 388 de 1997 se dio un paso fundamental para darle a la actividad del ordenamiento urbanístico el rango de una función pública, así como para 
modernizar la concepción y los instrumentos del planeamiento (Molina, 2003, p. 124)

Si bien con la Ley 9a se dio un importante paso en el proceso de consolidación del proceso de reforma urbana que el país requería, faltaba además que se elevara a rango constitucional el principio de que la propiedad del suelo es una función social, así como también de reglamentar el derecho de edificar sobre éste. Así entonces, en la nueva constitución de 1991 se consagró, por ejemplo, que "es deber del estado velar por la integridad del Espacio Público... las entidades públicas regularán la utilización del suelo y del espacio aéreo urbano en defensa del interés común...; cobrarán la plusvalía generada por la acción urbanística" (Artículo 82). En fin, la nueva constitución reforzaba la idea de que la práctica urbanística tuviera como soporte el hecho de que el "suelo" -y su "renta"- podían ser legítimamente "regulados" (Maldonado, 2003, p. 13)

Dados esos antecedentes, aparecería la ya mencionada LDT de 1997 que rige formalmente el ordenamiento territorial en Colombia en la actualidad, y que recogió en su momento principios, metodologías y experiencias de sistemas urbanísticos consolidados como los de España y Francia (Ramírez, 2011). Se pretendió con esa Ley darle a los instrumentos de PUR principios generales de orientación del desarrollo urbano y dotarlos de coherencia y jerarquía, en consonancia con los principios constitucionales anteriormente expuestos.

A partir de la expedición de la LDT, a todos los municipios colombianos se les impuso la tarea de elaborar sus respectivos POT, y bajo sus lineamientos regular el urbanismo físico o la práctica constructiva, es decir, en términos prácticos, el CU. De cierta manera, la nueva Ley le daría continuidad a la tradición local en PUR, sobre la cual se hacía mención al comienzo, de contar con dos tipos de niveles de planeamiento, uno general donde se plantea el MDU, y otro operativo, donde se interviene físicamente el espacio, por medio de una normativa específica de construcción de los AU.
Al respecto, Ezquiaga (2005) resalta sobre dichos niveles en la LDT "la relación dialéctica entre Ley y Plan, según la cual esta aporta una cobertura general pero son los Planes quienes deben concretarla y materializarla en un territorio determinado, requisito sin el cual la Ley no despliega su potencialidad" (18).

Dado lo anterior, se puede aquí plantear la idea de que el aprovechamiento urbanístico per se debe representar la materialización final de toda la cascada de planeamiento. De ahí se supone que midiendo los AU que está normados por el CU, se puede cuantificar hasta qué punto los principios discursivos de un MDU se ven reflejados en el desarrollo urbano d. A continuación entonces se estudia, en primer lugar, el contenido discursivo del POT de la ciudad de Sogamoso y su MDU, para luego simular en tres áreas los AU de acuerdo con la norma del CU, y medir algunas dimensiones de equidad espacial, sostenibilidad territorial y competitividad regional que se interpretan del modelo promulgado.

\section{El POT de Sogamoso: principios derivados del Modelo de Desarrollo Urbano Adoptado}

Sogamoso es una ciudad intermedia con una población proyectada para el año 2013 de 114.213 habitantes (DANE, 2005). Lo que la hace ser la segunda ciudad del departamento de Boyacá (Colombia), después de la capital Tunja (Figura 1). Tiene una extensión de 3.000 hectáreas, se localiza a $210 \mathrm{~km}$ de Bogotá y a $2.570 \mathrm{~m}$ de altitud sobre el nivel medio del mar. La vida económica de la ciudad combina actividades comerciales y de servicios en un 90\% (DANE, 2005), con algunas otras industriales relacionadas con la extracción de materias primas, esto es, el 9,5\% del total, según el mismo documento del DANE. Sogamoso es el segundo municipio que más actividad de carácter industrial ostenta en todo el departamento de Boyacá, superado levemente por el municipio cercano de Duitama pero por encima de la capital Tunja. 
Figura 1. El corredor comercial e industrial de la ciudad de Sogamoso.
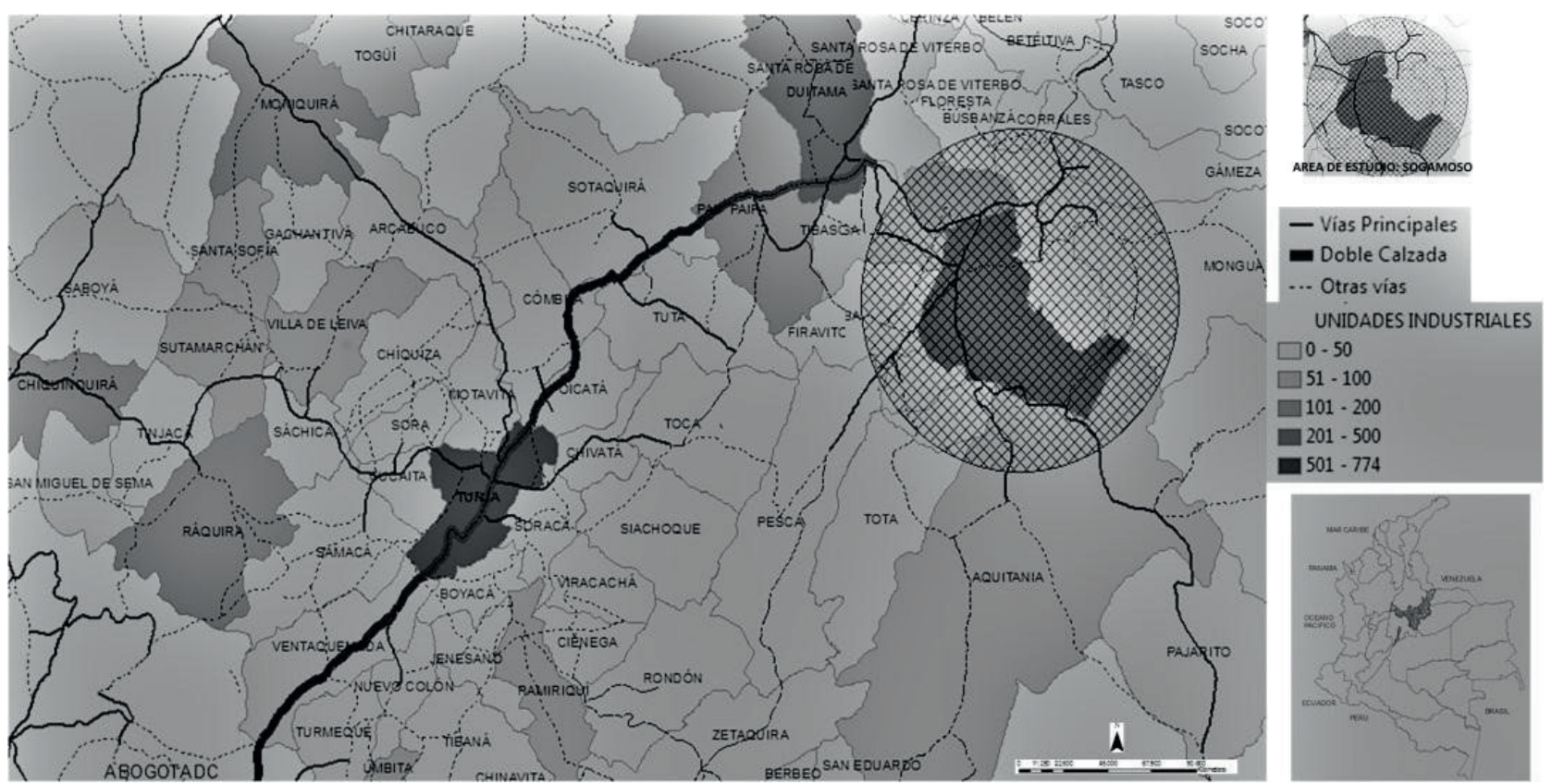

Fuente: elaboración propia a partir del SIG del IGAC (SIGAC, 2000)

Dicha configuración de la economía del municipio está asociada con algunos eventos que han marcado la evolución de su geografía económica, a saber: a. La línea del Ferrocarril del Nordeste en 1931 que conectó la ciudad con la dinámica económica nacional basada en exportaciones; b. la construcción a mediados del siglo XX de la planta siderúrgica Acerías Paz del Río, en el municipio cercano de Nobsa, a solo $6 \mathrm{~km}$ aproximadamente; c. y más recientemente la construcción de la doble calzada Bogotá - Sogamoso, la cual hace parte del proyecto de conexión trans-regional QuitoBogotá-Caracas.

La ciudad adoptó su POT en el año 2000 con el Acuerdo No. 096 del Concejo Municipal, y se mantiene hoy en día vigente como el documento oficial de Ordenamiento Territorial (a la fecha se encuentra en proceso de revisión ordinaria). En términos generales los objetivos del Plan se basan en el hecho primordial de que "Sogamoso es una ciudad de servicios de soporte fundamentalmente" (Concejo Municipal Sogamoso, 2000), y de los mismos se amplía la visión del MDU que es descrito así:
Ciudad regional de importancia nacional y eje del desarrollo provincial del nororiente colombiano, promoviendo integralmente la calidad devidadesushabitantes, imponiendo condiciones para su desarrollo, a través de la integración regional territorial, donde las variables de tamaño, mercado, y diversidad son comprendidas bajo las condiciones de capacidad para la competitividad. Optimización y racionalización de los recursos existentes como la infraestructura, los recursos naturales, los servicios sociales, la capacitación, y la inteligencia local como las condiciones para la competitividad; la calidad total en el hábitat, en el paisaje, en los servicios, sus coberturas y en las facilidades. Actitud política y social positiva para la participación democrática en el proyecto y propósito de desarrollo como el insumo esencial de la competitividad (Concejo Municipal Sogamoso, 2000, p. 7).

De dicho MDU se interpretan unos principios relacionados con la competitividad regional, la 
sostenibilidad territorial y la equidad espacial que se explican a continuación, y se señalan para cada uno de ellos unas dimensiones medibles asociadas a los mismos. Dichas dimensiones se relacionan de manera presumible con el Modelo, trayendo a colación conceptos semejantes extraídos de otras fuentes de literatura científica ${ }^{2}$. Lo que se hace posteriormente en cada una de las simulaciones de los AU es cuantificar la dimensión, es decir, calcular hasta qué punto, por ejemplo, el aprovechamiento es "diverso", lo cual en este caso es medido por el "porcentaje de área en usos diferentes al principal", y así sucesivamente para cada una de las dimensiones.

\section{Competitividad regional}

Cuando en la visión del MDU de Sogamoso se afirma que la ciudad se proyecta como "regional de importancia nacional y eje del desarrollo provincial del nororiente colombiano", ello se entiende como la capacidad de lograr que el carácter de nodo de la ciudad se convierta en una ventaja competitiva, y que por ello se faciliten las relaciones que las empresas locales establezcan con proveedores y otros agentes externos, configurando así cadenas productivas. Visto así el Modelo está en consonancia con el discurso global consistente en que las regiones deben transitar hacia la era de la economía de la información ${ }^{3}$, donde ellas ya no dependan tanto de las actividades y recursos con que cuentan localmente, sino además de las interrelaciones que las empresas logran desarrollar en su entorno más cercano (Yevenes \& Andalaft, 2005, p. 40), y por la concurrencia de diversas áreas productivas cuyas

\footnotetext{
$2 \quad$ Es un ejercicio deductivo.

3 Porter (1990) plantea que ya no son suficientes las ventajas comparativas (aquellas ventajas "naturales" que posee un estado. Generalmente no ha hecho nada especial para contar con este recurso) Ejemplo: El café suave en Colombia, la masiva mano de obra de la China, y que se hace necesario agregar valor a través de trabajo desarrollado previamente, generando nuevas ventajas en la oferta (diferenciación del producto y costos de producción).
}

conexiones permitan dar lugar a la generación de economías de aglomeración y externalidades, que hacen viable la especialización (Harmes-Liedtke, 2008; Isaza, 2010). De acuerdo con ello, en la Tabla 1 se proponen unas dimensiones y mediciones que cuantifican la pretensión de competitividad regional que se interpreta del $\mathrm{MDU}^{4}$.

\section{Sostenibilidad territorial}

Cuando se afirma en el MDU de Sogamoso que se busca la "optimización y racionalización de los recursos existentes"; ello se entiende como una pretensión de autosuficiencia, es decir, que para una misma oferta de servicios, se minimiza el consumo de recursos materiales, explotando al máximo los flujos locales, satisfaciendo el criterio de conservación de stocks y de reducción de residuos (Ministerio del Medio Ambiente [MMA], 1996). Para lograr dicha autosuficiencia, se deduce que el modelo tácitamente se basa en las bondades de la ciudad compacta, densa y policéntrica, en oposición a la ciudad dispersa, esto con el fin de hacer más complejas las interacciones urbanas, contener y cerrar los ciclos ecológicos, aumentar la eficiencia del suelo, garantizar la accesibilidad a los espacios libres y a las zonas verdes (Jiménez, 2011). En ese orden de ideas, la densidad, asociada a la compacidad, supone la generación de masas críticas interconectadas accesibles en su interior en función de la proximidad para ahorrar materia y energía, y a su exterior por sistemas eficientes de transporte público haciendo esencial la descentralización (Aguilera, 2008; Sabrino, 2005; Rodríguez, 2007). Es decir un policentrismo que no sobrepase los límites de su capacidad de carga soportada en nuevos sistemas de transporte e infraestructuras intermodales, y que evite razonablemente los

\footnotetext{
$4 \quad$ Se advierte que, aunque las mediciones no permiten validación alguna de las localizaciones en función de la renta del suelo, si es posible valorar la suficiencia de las localizaciones para atender las demandas derivadas del mercado que permitan materializar la pretensión de competitividad del MDU.
} 
Tabla 1. Mediciones propuestas para competitividad regional.

\begin{tabular}{|c|c|c|c|}
\hline Dimensión & Definición & Medición & Escala o parámetro* \\
\hline \multirow{2}{*}{$\begin{array}{l}\text { Estructura Urbana } \\
\text { Privada }\end{array}$} & \multirow{2}{*}{$\begin{array}{l}\text { Disponibilidad de suelo para el } \\
\text { mercado inmobiliario y productivo }\end{array}$} & \multirow[t]{2}{*}{ Porcentaje de área privada } & 0 (Mayor) \\
\hline & & & 100 (Menor) \\
\hline $\begin{array}{l}\text { Conectividad de } \\
\text { Usos Productivos }\end{array}$ & $\begin{array}{l}\text { Suficiencia de la articulación de } \\
\text { procesos de producción y servicios } \\
\text { complementarios a través de } \\
\text { infraestructuras viales. }\end{array}$ & $\begin{array}{l}\text { Relación de áreas dedicadas a la } \\
\text { movilidad por cada } \mathrm{m}^{2} \text { en usos } \\
\text { productivos. }\end{array}$ & $\begin{array}{l}0 \text { (menos conectada) } \\
\phi \\
\text { (más conectada) }\end{array}$ \\
\hline \multirow{2}{*}{$\begin{array}{l}\text { Umbral de } \\
\text { Demanda }\end{array}$} & \multirow{2}{*}{$\begin{array}{l}\text { Oportunidad de consumo, como de la } \\
\text { disponibilidad de mano de obra. }\end{array}$} & \multirow{2}{*}{$\begin{array}{l}\text { Relación de número de viviendas } \\
\text { por cada unidad Productiva. }\end{array}$} & 0 (Bajo) \\
\hline & & & 100 (Alto) \\
\hline $\begin{array}{l}\text { Especialización } \\
\text { Funcional }\end{array}$ & $\begin{array}{l}\text { Predominio de las actividades } \\
\text { económicas de un único sector. Es } \\
\text { decir se produce en términos relativos } \\
\text { mucho de un sector y poco de los } \\
\text { otros. }\end{array}$ & $\begin{array}{l}\text { Coeficiente de diversidad de } \\
\text { Shannon*** }\end{array}$ & $\begin{array}{l}0 \text { (especializado) } \\
(+-1)(\text { diverso })\end{array}$ \\
\hline \multicolumn{4}{|c|}{$\begin{array}{l}H^{\prime}=-\sum_{i=1}^{D} p_{i} \log _{2} p_{i}{ }^{*}-0 \\
\phi=\text { Infinito. La mayoría de las escalas son de } 0 \text { a infinito. También existen de infinito }\end{array}$} \\
\hline \multicolumn{4}{|c|}{$\begin{array}{l}\text { El índice de Shannon desarrollado para determinar la diversidad ecológica se utiliza aquí para estimar la } \\
\text { cantidad de sectores presentes en el área de estudio y la cantidad relativa de unidades de cada uno de esos } \\
\text { sectores (abundancia). Donde: } \mathrm{S} \text { - número de metros } 2 \text { por cada sector } \mathrm{Pi} \text { - proporción de Mt2 (i) respecto al total } \\
\text { (abundancia relativa del sector i): ni/N. ni - número de unidades del sector (i) N- número de todas las unidades de todos } \\
\text { las sectores }\end{array}$} \\
\hline
\end{tabular}

Tabla 2. Mediciones propuestas para sostenibilidad territorial.

\begin{tabular}{|c|c|c|c|}
\hline Dimensión & Definición & Medición & Escala o Parámetro \\
\hline Diversidad & La capacidad para propiciar la mezcla de usos. & $\begin{array}{l}\text { \% de área en usos } \\
\text { diferentes al principal. }\end{array}$ & $\begin{array}{l}0 \text { (menos diverso) } \\
100 \text { (más diverso) }\end{array}$ \\
\hline Compacidad & $\begin{array}{l}\text { Concentración de las estructuras funcionales } \\
\text { en configuraciones urbanas continúas, como } \\
\text { contraparte de la dispersión. }\end{array}$ & $\begin{array}{l}\text { Relación entre área } \\
\text { libre por cada metro } \\
\text { construido. }\end{array}$ & $\begin{array}{l}\Phi \text { (baja compacidad }) \\
-0 \text { (alta compacidad) }\end{array}$ \\
\hline Densidad & $\begin{array}{l}\text { Cantidad de individuos por unidad de superficie. } \\
\text { La densidad urbana puede referirse tanto a } \\
\text { habitantes como a edificaciones. }\end{array}$ & $\begin{array}{l}\text { Relación de unidades } \\
\text { construidas por } \\
\text { hectárea }\end{array}$ & $\begin{array}{l}0 \text { (baja densidad }) \\
\phi(\text { alta densidad })\end{array}$ \\
\hline Centralización & $\begin{array}{l}\text { Capacidad de la zona para proveer servicios. } \\
\text { Indicativo de nuevas centralidades } \\
\text { (estructuras poli céntricas). }\end{array}$ & $\begin{array}{l}\text { Relación de área de } \\
\text { equipamientos y } \\
\text { servicios por cada } \\
\text { unidad construida. }\end{array}$ & $\begin{array}{l}0 \text { (escasa centralidad) } \\
\phi(\text { alta centralidad })\end{array}$ \\
\hline $\begin{array}{l}\text { Espacio público } \\
\text { per-cápita }\end{array}$ & $\begin{array}{l}\text { Capacidad para generar un entorno favorable a } \\
\text { las ofertas culturales y lúdicas que incrementen } \\
\text { la interacción social. }\end{array}$ & $\begin{array}{l}\text { Relación de Área } \\
\text { de espacio público } \\
\text { recreativo (parques, } \\
\text { plazas, etc.) por } \\
\text { habitante. }\end{array}$ & $\begin{array}{l}0 \text { (menor) } \\
\phi \text { (mayor) }\end{array}$ \\
\hline
\end{tabular}

Fuente: elaboración propia. 
problemas ligados a la compacidad tales como usos altamente incompatibles, invasión de lo público, pocas disponibilidades de suelo para usos poco rentables, congestión vehicular, entre otras. De acuerdo con esto, en la Tabla 2 se proponen unas dimensiones que se consideran representan la pretensión de sostenibilidad territorial que se deriva del Modelo.

\section{Equidad espacial}

Cuando en el MDU de Sogamoso se afirma que se busca "...la calidad total en el hábitat, en el paisaje, en los servicios, sus coberturas y en las facilidades", ello se entiende fundamentalmente como el acceso justo y equitativo a los servicios urbanos para la satisfacción de las necesidades. Si por justicia se entiende dar a cada cual lo que se merece y por equidad ofrecer iguales condiciones a todos, se deduce que el modelo se orienta a proveer condiciones para que el acceso, los beneficios y males fueran similares para todos, de modo que no existan diferencias en las ventajas y limitaciones de acceso de la población a los servicios, y donde el impacto potencial esté repartido de forma sensiblemente similar entre lugares y personas (Bosque, Díaz \& Díaz, 2002). Por tanto, del modelo se supone la generación de suelo urbanizado articulado funcionalmente a la ciudad, dotado de proporciones adecuadas de espacio público y libre, el cual proporcione calidad al entorno, y una regulación urbanística orientada a proveer el acceso justo y equitativo de los servicios urbanos y la configuración de las actividades, tendiente a facilitar la interacción y por tanto a reducir la segregación residencial socio-espacial y la segregación de acceso socioeconómica. En la Tabla 3 se proponen unas dimensiones mediciones que se considera representan la pretensión de equidad espacial que se deriva del Modelo.

\section{Metodología}

\section{Selección de las áreas y generalidades de la simulación tridimensional}

Luego de la promulgación del POT de Sogamoso y de su MDU, se estableció por medio del Decreto No. 203 del 11 de diciembre de 2002, el CU que busca operacionalizar el Modelo planteado, y que reglamenta el conjunto de normas de urbanismo y construcción en suelo urbano y de expansión urbana, definiendo el alcance de los AU. Se seleccionaron tres zonas que fueron los espacios sometidos a simulaciones tridimensionales de acuerdo con las indicaciones normativas del CU (Figura 2).

Tabla 3. Mediciones propuestas para equidad espacial.

\begin{tabular}{|c|c|c|c|}
\hline Dimensión & Definición & Medición & Escala o Parámetro \\
\hline Accesibilidad & $\begin{array}{l}\text { Facilidad para la provisión de los } \\
\text { servicios urbanos. }\end{array}$ & $\begin{array}{l}\text { Distancia media de las } \\
\text { áreas residenciales a los } \\
\text { equipamientos locales. }\end{array}$ & $\begin{array}{l}0 \text { (Próxima) } \\
\phi \text { (Lejana) }\end{array}$ \\
\hline $\begin{array}{l}\text { Calidad del } \\
\text { Entorno }\end{array}$ & $\begin{array}{l}\text { Condición para la gratificación } \\
\text { proporcionada por el paisaje } \\
\text { urbano. }\end{array}$ & $\begin{array}{l}\text { Relación de número de } \\
\text { unidades edificadas por cada } \\
\text { Hectárea de espacio público. }\end{array}$ & $\begin{array}{l}0 \text { (Mayor } \\
\text { calidad) } \phi \\
\text { (Menor calidad) }\end{array}$ \\
\hline Balance de cargas & $\begin{array}{l}\text { Distribución de cargas urbanísticas } \\
\text { entre los desarrolladores privados y } \\
\text { la ciudad. }\end{array}$ & $\begin{array}{l}\text { Relación de cesiones sistemas } \\
\text { generales construida }\left(\mathrm{m}^{2}\right) \\
\text { públicas para por área. }\end{array}$ & $\begin{array}{l}0 \text { (Favorable al } \\
\text { propietario) } \phi \\
\text { (favorable a la } \\
\text { ciudad) }\end{array}$ \\
\hline
\end{tabular}

Fuente: elaboración propia. 
Figura 2. Localización de las áreas simuladas y comparadas por medio de las mediciones.

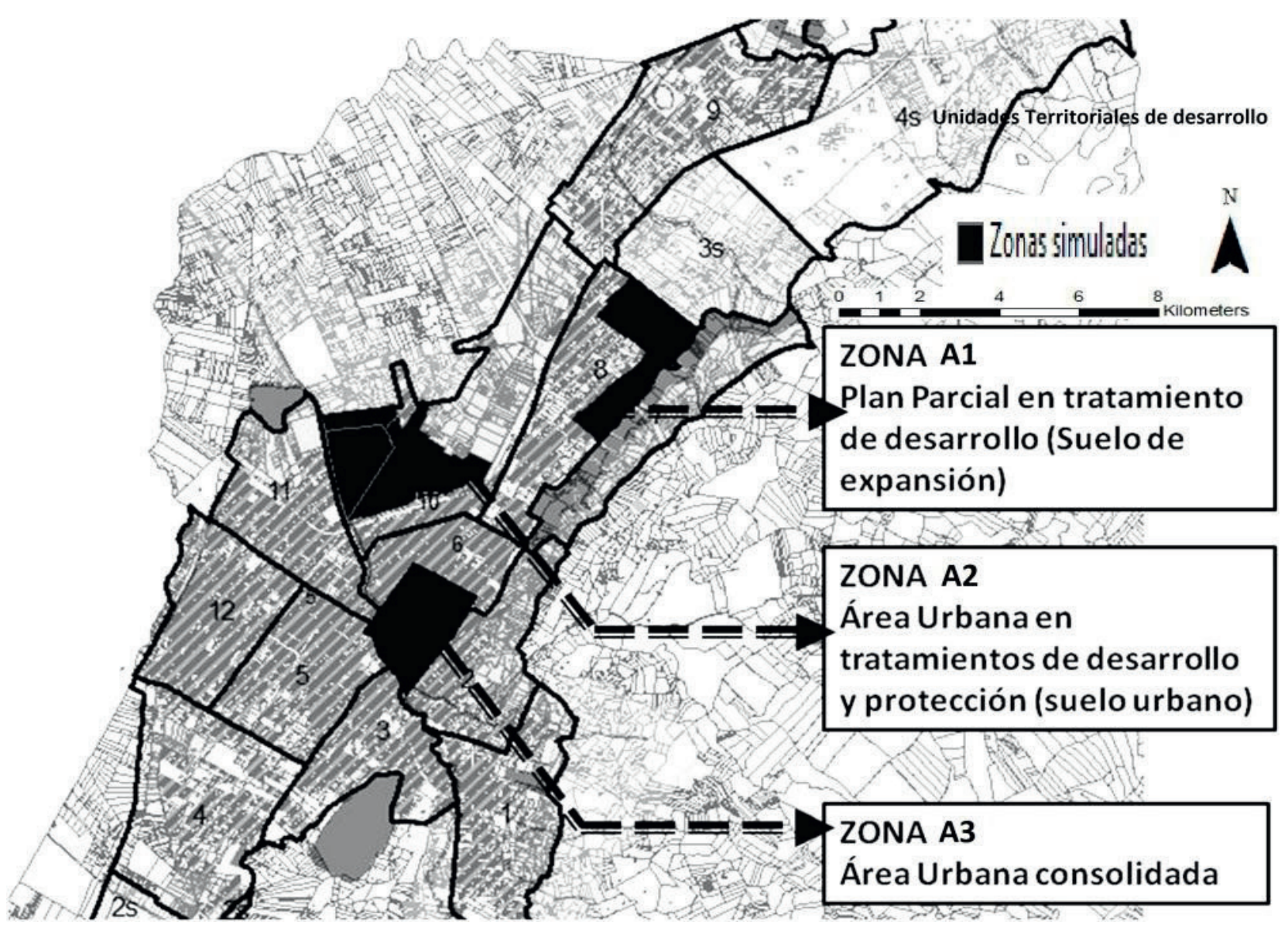

Fuente: elaboración propia a partir del SIG del IGAC (SIGAC, 2000).

La zona A1 cuenta con un área de aproximadamente 50 hectáreas, y se sitúa en los límites de una ladera profusamente explotada por la minería del carbón y sílice para las industrias cementera, ladrillera y de acerías. Esta zona fue definida por el POT como área de expansión urbana, por tanto con la obligatoriedad de formular Planes Parciales para su desarrollo. Éstos fueron adoptados finalmente en el año 2006 con el propósito de desarrollar proyectos de vivienda de interés social.

La zona A2 cuenta con unárea de aproximadamente de 76.7 hectáreas en el interior de perímetro urbano, de las cuales 21,98 hectáreas corresponden a un área del Barrio Jorge Eliécer Gaitán declarado con tratamiento de protección para la conservación de humedales, con condiciones desfavorables y algunas restricciones para ser urbanizado. A las áreas restantes el POT les asignó un tratamiento de desarrollo, manteniendo su vocación residencial. Actualmente la zona está delimitada por la vía regional de acceso a la doble calzada que conecta a Bogotá y al sur por la vía férrea, y cuenta con 498 edificaciones con alturas que oscilan entre uno y siete pisos, con predominio de vivienda unifamiliar.

La zona A3 corresponde al centro fundacional, económico y administrativo de Sogamoso. La actividad dominante es el comercio de bienes y servicios, aunque aún conserva una fuerte actividad residencial. Esta zona incorpora según el POT los tratamientos de consolidación urbanística, renovación y redesarrollo. 
Figura 3. Secuencia del proceso de simulación tridimensional.

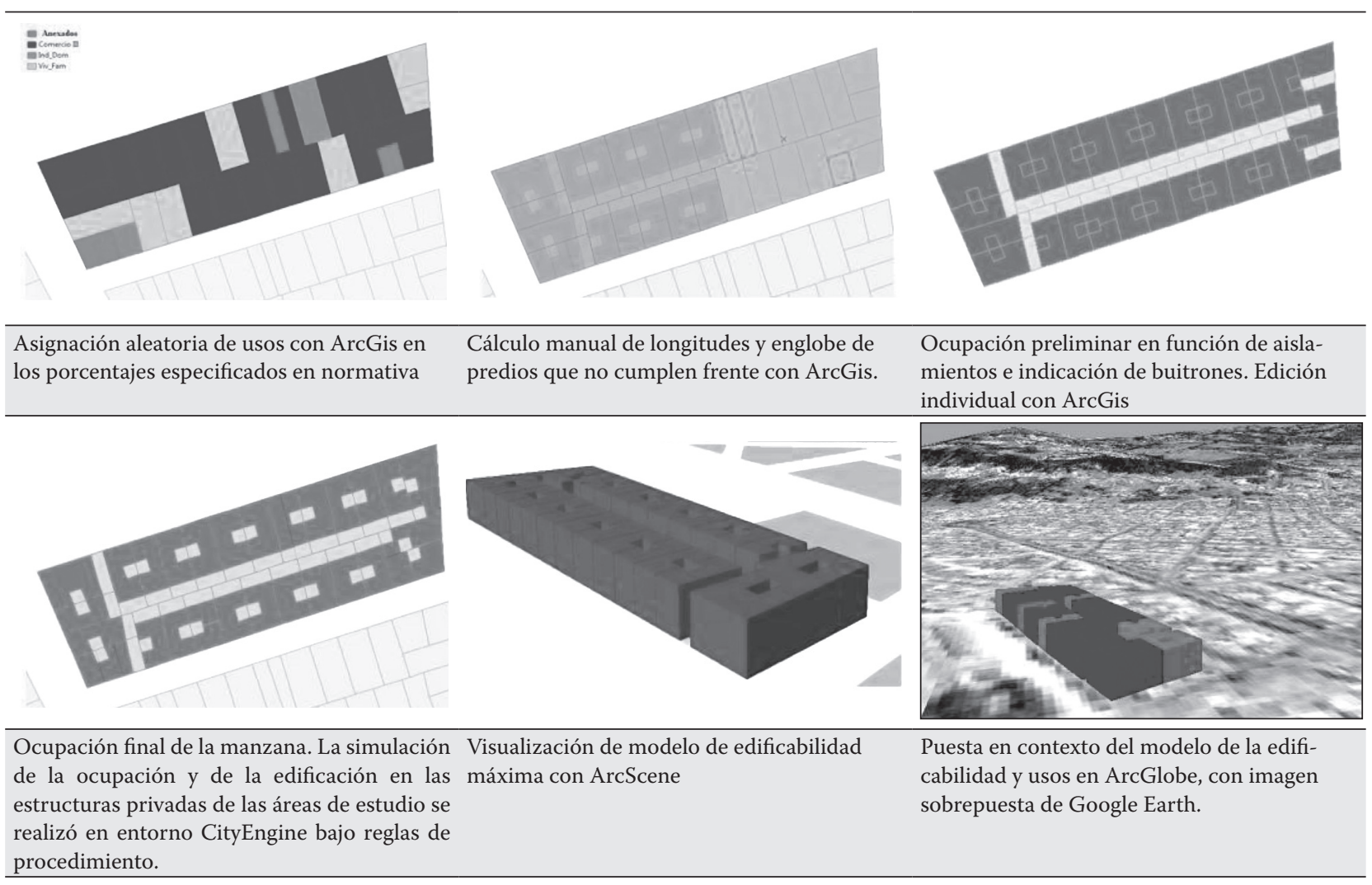

Sobre dichas tres zonas se hicieron las respectivas simulaciones tridimensionales ${ }^{5}$. Las simulaciones utilizaron el software de manera complementaria y requirieron de dos vías de ensamble. Por una parte en un ambiente SIG se procesaron los mapas de clasificación del suelo, áreas de actividad, instrumentos de planificación y proyectos relevantes en las Unidades Territoriales y, por otra, la vinculación de las fichas normativas del $\mathrm{CU}$ a las propiedades prediales, cuyo resultado es el mapa de edificabilidad que fue sometido a los procedimientos que ilustra la Figura 3, a partir de una selección de prototipos de desarrollo urbanístico, y de unos criterios de optimización de

5 Para la realización de las respectivas simulaciones se utilizó una combinación de las aplicaciones ArcGis y ArcScene, para los montajes de las imágenes se utilizaron Sketchup y Google Earth, y para la mayor parte del modelamiento final se usó CityEngine versión 12. la edificabilidad y de los usos, desde la perspectiva del máximo aprovechamiento inmobiliario permitido por el CU. El proceso metodológico que incorpora las entradas de datos para secuencia: modelación-simulación y cálculo se muestra en la Figura 4.

Se hizo un primer modelamiento de lo que el POT diagnosticó estaba construido en cada una de esas zonas. El segundo modelamiento corresponde a la simulación que proyecta espacialmente los AU en sujeción a la normativa que determina para las mismas zonas el CU del municipio, de acuerdo con unos prototipos normativos expuestos en la Tabla 4, y que reflejan las principales variantes de cada ficha normativa contemplada en el mismo código. Luego de simular las tres áreas, se aplicaron las respectivas mediciones sobre los arquetipos simulados. A continuación se detallan los resultados obtenidos. 
Figura 4. Proceso metodológico.



Tabla 4. Muestra de los principales prototipos edilicios derivados del Código Urbano

\begin{tabular}{|c|c|}
\hline \multirow[t]{10}{*}{ Prototipo } & Indicación Normativa (Fichas) \\
\hline & Densidad: Controlada" \\
\hline & Volumetría: Aislado*: \\
\hline & Índices de ocupación y contrucción: 70\%-140\% \\
\hline & Altura en número de pisos: 2 \\
\hline & Área mínima de lote: $100 \mathrm{~m} 2$ \\
\hline & Aislamiento posterior: $3 \mathrm{~m}$ \\
\hline & Longitud mínima de fachada: $6 \mathrm{~m}$ \\
\hline & Uso principal: Residencial \\
\hline & Usos posibles: $\mathrm{M}, \mathrm{C}, \mathrm{IS}, \mathrm{A}$ * \\
\hline & Uso prohibido: Industrial \\
\hline & Densidad: Autoregulable $* *: * * *$ \\
\hline & Volumetría: Pareado \\
\hline & Índices de ocupación y construcción: Resultante \\
\hline & Altura en número de pisos: 2 \\
\hline & Área mínima de lote: $100 \mathrm{~m} 2$ \\
\hline & Aislamiento posterior: $3 \mathrm{~m}$ \\
\hline & Aislamiento lateral 0 \\
\hline & Longitud mínima de fachada: $6 \mathrm{~m}$ \\
\hline & Uso principal: M.C \\
\hline & Usos posibles: $\mathrm{R}$. \\
\hline & Uso prohibido: Industrial \\
\hline
\end{tabular}




\begin{tabular}{l}
\multicolumn{1}{c}{ Indicación Normativa (Fichas) } \\
\hline $\begin{array}{l}\text { Densidad: Resultante } \\
\text { Volumetría: Mixta }\end{array}$ \\
$\begin{array}{l}\text { Índices de ocupación y construcción: Resultante } \\
\text { Área mínima de lote: } 100 \mathrm{~m}\end{array}$ \\
$\begin{array}{l}\text { Aislamiento posterior: } 6 \mathrm{~m} \\
\text { Aislamiento lateral: } 0\end{array}$ \\
$\begin{array}{l}\text { Longitud mínima de fachada: } 8 \mathrm{~m} \\
\text { Uso principal: } \mathrm{R}\end{array}$ \\
\begin{tabular}{l} 
Usos posibles: $\mathrm{M}$, C, IS, $\mathrm{A}$ \\
\hline Uso prohibido: Industrial
\end{tabular} \\
$\begin{array}{l}\text { Área mínima del buitrón } * * * * * * *: 20 \mathrm{~m} 2 \\
\text { Longitud mínima buitrón: } 5 \mathrm{~m}{ }^{2}\end{array}$ \\
\hline
\end{tabular}

Fuente: elaboración propia

*Esta categoria corresponde a áreas de tratamiento especial, donde es inadecuada la utilización intensiva del suelo y por lo tanto deben controlarse los asentamientos, (35\% del área bruta urbanizable, para cesión tipo A en suelo urbano y de expansión urbana). ${ }^{* * *}$ Las volumetrías se explican así en la norma, a. aislada: se presenta cuando entre edificaciones existe un aislamiento predeterminado; b. pareada: se presenta cuando los MUDos resultantes de las construcciones están separados por la junta de dilatación y se conjugan en forma similar el manejo de las alturas; c. Mixta: se presentan cuando se conjugan las culatas aisladas y pareadas en altura. **** Convenciones de usos: $\mathrm{M}=$ uso mixto; $\mathrm{C}=$ uso comercial; IS=Uso institucional; $\mathrm{R}$ Uso residencial; $\mathrm{A}=\mathrm{uso}_{\text {agropecuario }}$ ****: Es la categoría correspondiente a las áreas que aún no se han desarrollado, o lo han hecho en forma incipiente, donde se hacen necesaria una utilización del suelo de alta o mediana intensidad, por lo cual se exigen mínimo 45 viviendas por hectárea bruta urbanizable, para nuevas urbanizaciones y agrupaciones. ${ }^{* * * * * *}$ es la resultante de aplicar los aislamientos y otras normas que regulan la ocupación y el volumen, pues el POT solo en unos pocos casos hace explícitos numéricamente estos índices. ${ }^{* * * * * * * * * *}$ Los buitrones son vacios del volumen edificado para iluminar y ventilar espacios.

\section{Resultados de las mediciones y comparaciones}

\section{Resultados para la Competitividad Regional}

De acuerdo con la Tabla 5, la media urbana indica que hay un ligero crecimiento de los valores obtenidos para la estructura urbana privada en la proyección frente a lo que sucede en la realidad. El margen es indicio de que existe un mayor atractividad para la inversión inmobiliaria, es decir, una mayor capacidad local para atraer y retener poblaciones o inversiones. Sin embargo, habría que matizar su alcance de acuerdo al porcentaje de áreas vacantes por desarrollar. Respecto a la conectividad de los usos productivos, se evidencia una ligera mejora para el necesario encadenamiento productivo que se supone debe darse para cumplir este principio del modelo. El umbral de demanda cae dramáticamente en los arquetipos proyectados de todas las zonas, en especial en la zona A2 de suelo urbano, lo que pone en duda la disponibilidad de mano de obra o de futuros consumidores de las actividades productivas. Finalmente, la zona central A3 privilegia en la simulación proyectada usos diferentes a los residenciales, en tanto estos son acogidos por las nuevas áreas desarrolladas que mantienen un balance favorable a la diversificación productiva, lo cual se deriva de la limitación de la norma.

\section{Resultados para la Sostenibilidad Territorial}

De acuerdo con la Tabla 6 las mediciones expresan que la diversidad aumenta proporcionalmente según el grado de consolidación de la zona estudiada, es decir, es mayor en la zona 
Tabla 5. Mediciones comparadas de competitividad regional.

\begin{tabular}{lcccccccc}
\hline \multicolumn{1}{c}{$\begin{array}{c}\text { Dimensión } \\
\text { Medida }\end{array}$} & $\begin{array}{c}\text { ZA1 } \\
\text { Actual }\end{array}$ & $\begin{array}{c}\text { ZA1 } \\
\text { Proyec. }\end{array}$ & $\begin{array}{c}\text { ZA2 } \\
\text { Actual }\end{array}$ & $\begin{array}{c}\text { ZA2 } \\
\text { Proyec. }\end{array}$ & $\begin{array}{c}\text { ZA3 } \\
\text { Actual }\end{array}$ & $\begin{array}{c}\text { ZA3 } \\
\text { Proyec. }\end{array}$ & $\begin{array}{c}\text { Promedio } \\
\text { Actual }\end{array}$ & $\begin{array}{c}\text { Promedio } \\
\text { Proyec. }\end{array}$ \\
\hline $\begin{array}{l}\text { Estructura Urbana } \\
\text { Privada }\end{array}$ & 69,76 & 53,98 & 56,97 & 38,48 & 69,66 & 64,39 & $\mathbf{6 5 , 4 6}$ & $\mathbf{5 2 , 2 8}$ \\
\hline $\begin{array}{l}\text { Conectividad de Usos } \\
\text { Productivos }\end{array}$ & 0,16 & 0,72 & 0,84 & 0,34 & 0,46 & 0,52 & $\mathbf{0 , 4 9}$ & $\mathbf{0 , 5 3}$ \\
\hline Umbral de demanda & 8,25 & 0,96 & 17,37 & 0,72 & 0,33 & 0,25 & $\mathbf{8 , 6 5}$ & $\mathbf{0 , 6 4}$ \\
\hline Especialización Funcional. & 0,71 & 1,36 & 0,61 & 1,13 & 1,05 & 1,22 & $\mathbf{0 , 7 9}$ & $\mathbf{1 , 2 4}$ \\
\hline
\end{tabular}

centro A3. Igualmente es evidente una reducción proporcional del espacio público, y un dramático incremento en la densidad. Dicha tendencia es más significativa donde hay aumentos en los $\mathrm{AU}$, que indican naturalmente una mayor compacidad asociada a las densidades. La capacidad para centralizar funciones se incrementa al crecer el número de equipamientos, lo cual es más visible en la zona $\mathrm{A} 2$, con tratamiento de desarrollo, lo que implica la posibilidad de nuevas centralidades, pero hay una notable reducción del espacio público per cápita, que se explica porque las zonas estudiadas incorporan un mayor número de hogares, y la aplicación de la norma de cesiones es restringida, excepto en el caso de la zona A2 donde existe una amplia zona de protección ambiental. Por ello, en la dimensión "espacio público" solo la simulación en zona A2 cumple la meta nacional de $15 \mathrm{~m}^{2}$ por persona según el CONPES 3718, pero las otras zonas están lejos de lograrla.

Los resultados muestran que las tres zonas simuladas se diversifican (lo que está en consonancia con la medición anterior de reducción de la especialización funcional), aumenta la capacidad para centralizar funciones y hay notables incrementos en la densidad que reflejan una mayor compacidad, condiciones que en conjunto hacen más compleja la interacción, posibilitando unos ámbitos urbanos que propician la sostenibilidad promulgada en el Modelo. Pero esas densidades tienen sus límites y Sogamoso los transgrede si se observa el promedio simulado al propiciar una escasa generación de nuevo espacio público, una reducción relativa del espacio libre así como

Tabla 6. Mediciones comparadas de equidad espacial.

\begin{tabular}{|lrrrrr|r|r|r|}
\hline \multicolumn{1}{c}{$\begin{array}{c}\text { Dimensión } \\
\text { Medida }\end{array}$} & $\begin{array}{c}\text { Z1 } \\
\text { Actual }\end{array}$ & $\begin{array}{c}\text { Z1 } \\
\text { Proyec }\end{array}$ & $\begin{array}{c}\text { Z2 } \\
\text { Actual }\end{array}$ & $\begin{array}{c}\text { Z2 } \\
\text { Proyec }\end{array}$ & $\begin{array}{c}\text { Z3 } \\
\text { Actual }\end{array}$ & $\begin{array}{c}\text { Z3 } \\
\text { Proyec }\end{array}$ & $\begin{array}{c}\text { Promedio } \\
\text { Actual }\end{array}$ & $\begin{array}{c}\text { Promedio } \\
\text { Proyec }\end{array}$ \\
\hline \multicolumn{2}{c}{ Centro } & \multicolumn{3}{c}{$\begin{array}{c}\text { Tratamiento de } \\
\text { Desarrollo }\end{array}$} & \multicolumn{2}{c}{ Plan Parcial } & \multicolumn{2}{c|}{ Media Urbana } \\
\hline Diversidad & 22.8 & 26.31 & 15.56 & 37.48 & 31.72 & 47.25 & $\mathbf{2 3 . 3 6}$ & $\mathbf{3 7 . 0 1}$ \\
\hline Compacidad & 30.34 & 0.19 & 7.02 & 2.98 & 0.45 & 0.26 & $\mathbf{1 2 . 6}$ & $\mathbf{1 . 1 4}$ \\
\hline Densidad & 12.29 & 285.77 & 6.49 & 38.81 & 29.54 & 245.36 & $\mathbf{1 6 . 1 1}$ & $\mathbf{1 8 9 . 9 8}$ \\
\hline Centralización & 127.9 & 3.12 & 18.17 & 13.1 & 15.96 & 5.29 & $\mathbf{5 4 . 0 1}$ & 7.17 \\
\hline Espacio Público & 67.47 & 1.96 & 3.78 & 18.66 & 0.82 & 0.78 & $\mathbf{2 4 . 0 2}$ & 7.13 \\
\hline
\end{tabular}

Fuente: elaboración propia. 
Tabla 7. Mediciones comparadas de equidad espacial.

\begin{tabular}{lcccccccc}
\hline $\begin{array}{c}\text { Dimensión } \\
\text { Medida }\end{array}$ & $\begin{array}{c}\text { ZA1 } \\
\text { Actual }\end{array}$ & $\begin{array}{c}\text { ZA1 } \\
\text { Proyec }\end{array}$ & $\begin{array}{c}\text { ZA2 } \\
\text { Actual }\end{array}$ & $\begin{array}{c}\text { ZA2 } \\
\text { Proyec }\end{array}$ & $\begin{array}{c}\text { ZA3 } \\
\text { Actual }\end{array}$ & $\begin{array}{c}\text { ZA3 } \\
\text { Proyec }\end{array}$ & $\begin{array}{c}\text { Promedio } \\
\text { Actual }\end{array}$ & $\begin{array}{c}\text { Promedio } \\
\text { Proyec }\end{array}$ \\
\hline Accesibilidad & 142,94 & 47,43 & 136,54 & 76,74 & 51,45 & 69,9 & $\mathbf{1 1 0 , 3 1}$ & $\mathbf{6 4 , 6 9}$ \\
\hline Calidad del entorno & 12,96 & 620,95 & 84,87 & 63,08 & 392,78 & 688,96 & $\mathbf{1 6 3 , 5 4}$ & $\mathbf{4 5 7 , 6 6}$ \\
\hline Balance de cargas & 7,69 & 0,16 & 3,17 & 1,99 & 0,25 & 0,15 & $\mathbf{3 , 7}$ & $\mathbf{0 , 7 7}$ \\
\hline
\end{tabular}

Fuente: elaboración propia.

su fraccionamiento. Además, la autocontención espacial de los flujos ecológicos, a costa de la calidad de los entornos urbanos, resulta contraproducente, pues limita la fluidez, de la estructura ecológica, donde la continuidad del espacio libre y del público son esenciales, acelera el deterioro obligando al traslado de residentes y limita su papel como barrera al agudo problema de emisiones en una ciudad de carácter industrial.

\section{Resultados para Equidad Espacial}

De acuerdo con la Tabla 7, la zona A1 que representa la localización de los hogares en las áreas perimetrales de la ciudad es la que alcanza más accesibilidad en la proyección. Esto puede resultar paradójico, sin embargo ocurre porque el mayor número de equipamientos se traduce en menores distancias a la vivienda, aunque este indicador no expresa suficientemente las dificultades que entraña el acceso a las mismas, pues para definirlos el modelo se apoyó en una distribución teórica porcentual y des localizada de los usos, y en las especificaciones normativas para las nuevas urbanizaciones que obligan a un porcentaje de equipamientos comunitarios pero sin especificarlos.

Por otra parte, la calidad del entorno baja dramáticamente en la zona A1 y en la zona A3, excepto la zona A2 que cuenta con una reserva. Mientras tanto, en todos los casos, el balance de cargas se vuelve favorable a los propietarios. Esta medida es tal vez la que genera más preocupaciones, ya que evidencia cómo el sector público es quien termina responsabilizándose al final, de las obras de urbanismo y de espacio público de mayor impacto. Como el crecimiento del número de construcciones es exponencial en las tres áreas proyectadas, esto exige un mayor número de equipamientos y sistemas generales, requiriendo mayores cargas, pero estas cesiones no son asumidas por todas las unidades construidas, trasladándolas más bien a la ciudad como un conjunto (Figura 5).

Figura 5. Porcentaje de participación de áreas urbanizadas, de desarrollo individual predio a predio y de espacio público por rango de tamaño de parcela en el área con tratamiento de desarrollo.

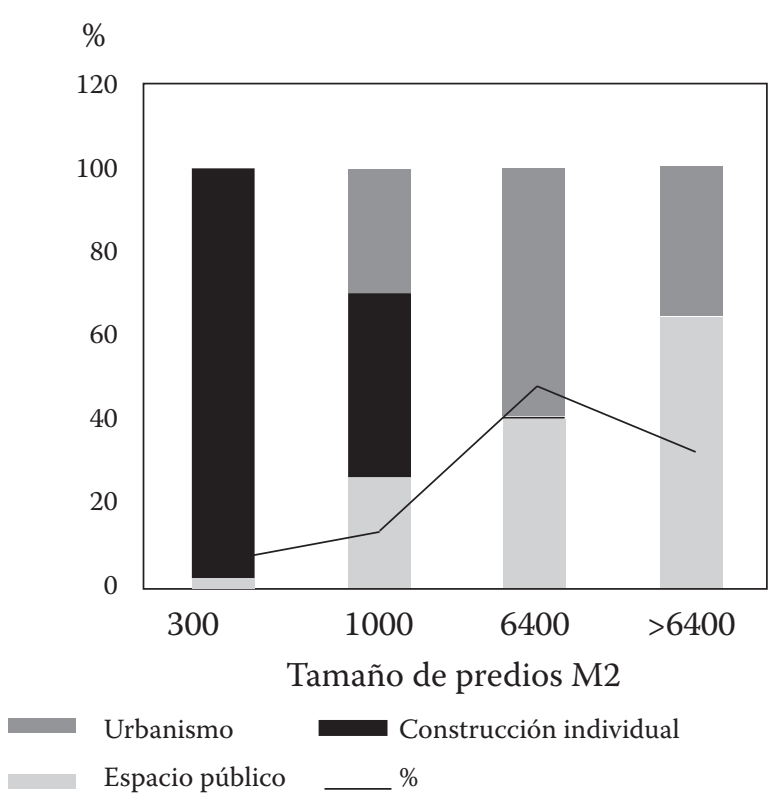

Fuente: elaboración propia. 
Lo anterior se evidencia claramente con los Planes Parciales para la zona A1, donde los parques urbanos deben ser enajenados, financiados y gestionados directamente por la municipalidad al desligarlos de las cargas a que se obligarían los promotores y propietarios del suelo, a condición de hacer viables algunos de los emprendimientos. Eso mismo sucede con las áreas de protección declaradas en la zona A2. Este desbalance tiene su explicación en la generalización de las excepciones normativas, que en la práctica revalida el urbanismo "predio a predio", y a la laxitud con que ellos se reglamentan al no establecer parámetros diferentes de la localización y enunciar su tratamiento, haciendo inocuas las definiciones sobre el reparto equitativo de cargas y beneficios. Si esto sucede en áreas con tratamientos de desarrollo, también hay una contribución pobre de las nuevas urbanizaciones en áreas consolidadas o en crecimiento, donde debido al tamaño de los lotes vacantes o los que no alcanzan los topes de los $\mathrm{AU}$, se construye parcela a parcela.

\section{Conclusiones}

Se ha visto que los $\mathrm{AU}$, concretan los volúmenes y actividades que configuran la ciudad con base en los lineamientos generales del POT, y cumpliendo una normativa específica del $\mathrm{CU}$, lo que permite materializar un MDU. Si bien el MDU del POT de Sogamoso pretende balancear en el discurso general las pretensiones propias de la competitividad, sostenibilidad y equidad en el territorio, la proyección simulada de los AU reglamentados por el CU, muestra que existen de entrada incompatibilidades entre dichas pretensiones. Al declararse como un Modelo compacto y derivar unas mayores densidades, efectivamente la simulación proyectada del aprovechamiento expresa una mayor compacidad que favorece la autosuficiencia de los flujos, sin embargo la proporción edificada frente al espacio público liberado dificulta un entorno de calidad. Por otra parte, el incremento de unidades edificadas por hectárea de espacio público coincide con altas densidades y propicia espacios insalubres. El incremento en usos diferentes al principal en adición a la medición de la diversidad, muestra que la materialización del Modelo es proclive a la diversidad, pero con un desbalance entre volúmenes edificados y espacios libres y públicos, es decir, en detrimento de la calidad del paisaje. La capacidad para centralizar funciones aumenta en todos los casos, lo que confirma que potencialmente podrían derivarse nuevas centralidades, y expresa la pretensión policéntrica del mismo Modelo.

Cuando se aplica la normativa derivada del CU en las proyecciones simuladas, se refleja que el principio de sostenibilidad territorial del MDU se cumple, dada cierta autonomía y equilibrio en el acceso de los servicios urbanos vitales, no obstante se generan algunas diferencias en términos de la conectividad a los equipamientos productivos. Se muestra en todos los casos una progresiva diversificación de los usos, lo cual confirma la pretendida especialización productiva del centro proyectado, con un leve incremento de la estructura privada. Las distancias medias a los equipamientos se reducen casi a la mitad, especialmente en áreas periféricas. Eso significa una sustancial mejora en la distribución urbana de los equipamientos locales y mayor autonomía de las diferentes zonas de la ciudad para prestar los servicios urbanos básicos. Esta observación deriva exclusivamente de las distancias medias a los equipamientos que solo en el centro se amplía, porque a partir de allí, existiendo mayor número de unidades de servicios para las viviendas bajo la norma, se reducen las distancias y eso induce a pensar en una mejor distribución espacial.

Mientras todas las dimensiones de los principios de competitividad y equidad del MDU se afirman hasta cierto punto en las proyecciones, el reparto de cargas y beneficios como una dimensión importante del principio de equidad espacial se niega sistemáticamente. La simulación muestra que, en todas las zonas estudiadas, Sogamoso se obliga a trasladar a toda la ciudad parte de las cargas que debiera asumir el promotor o el propietario del 
suelo para viabilizar financieramente los planes parciales, los tratamientos de desarrollo o de consolidación; introduciendo un fuerte factor de inequidad en el desarrollo de la ciudad. Esto a pesar de la supuesta primacía de este principio del discurso sobre los demás.

El ejercicio de medición anteriormente resumido permite considerar que la valoración del éxito o fracaso de la aplicación de políticas de ordenamiento territorial requiere la estandarización de metodologías que permitan evaluaciones más precisas de su alcance e impacto. La complejidad de los instrumentos de PUR no solo se hace evidente al momento de su formulación, sino también de su seguimiento y evaluación. No solo basta que se formule un excelente MDU en un POT, sino que se requiere la certeza de que la mayoría de sus principios perduren y sean respetados en la etapa de aplicación de la norma urbana contenida en los CU. De lo contrario, poco o nada se habrá avanzado en el intento de salir de la trampa del urbanismo precario y desarticulado, llamado del predio a predio, y el cual se pretendió superar precisamente con la formulación de la Ley 388 de 1997.

\section{Referencias bibliográficas}

Aguilera, F. (2008). "Análisis espacial para la ordenación eco-paisajística de la aglomeración urbana de Granada". Disertación Doctoral no publicada, Universidad de Granada, Andalucía, España.

Bosque, J.; Díaz, C.; Díaz, M. A. (2001-2002). “De la justicia espacial a la justicia ambiental en la política de localización de instalaciones para la gestión de residuos en la comunidad de Madrid". Boletín de la Real Sociedad Geográfica, 137-138, 89-114. Recuperado de: [http://www.realsociedadgeografica.com/es/site/boletin.asp].

Concejo Municipal de Sogamoso. Acuerdo No. 096 de 2000. Por el cual se adopta el Plan de Ordenamiento Territorial del municipio de Sogamoso - Boyacá y se conceden unasfacultades. Recuperado de: [http://www.sogamoso-boyaca.gov.co/apc-aafiles/65616664353566303162646663663062/ acuerdo_096_del_2000_adopcion_del_pot.pdf].
Departamento Administrativo Nacional de Estadística. (2005). Boletín Censo General 2005. Perfil Sogamoso-Boyacá. Recuperado de: [http://www. dane.gov.co/files/censo2005/perfiles/boyaca/ sogamoso.pdf].

Departamento Administrativo Nacional de Estadística. (2005). Estimación y proyección de población nacional, departamental y municipal total por área 1985-2020. Recuperado de: [http:// www.dane.gov.co/index.php/poblacion-y-demografia/proyecciones-de-poblacion].

ESRI (2012) CityEngine 2012 Software. Enviromental Systems Research Institute. Redland. USA.

ESRI (2011) ArcGis 10.1 Software. Enviromental Systems Research Institute. Redland. USA.

Ezquiaga, J. M. (2005). Patrones urbanísticos y arquitectónicos dirigida a sectores de bajos ingresos en Bogotá. Prólogo. Tarchópulos D. \& Ceballos O. Bogotá: Universidad Javeriana.

Harmes-Liedtke, U. (2008). "Benchmarking territorial competitivo". Recuperado de: [http://www. slideshare.net/iorifoar/benchmarking-territorialcompetitivo].

Isaza, J. (2010). “Cadenas productivas. Enfoques y precisiones conceptuales". Sotavento, (11), pp. 8-25. Recuperado de: [http://foros.uexternado. edu.co/ecoinstitucional/index.php/sotavento].

Jiménez, L (2010) "Alternativas a la dispersión urbana. Análisis de indicadores basados en nuevas estrategias para el desarrollo sostenible". Tesis de Master en Gestión y Valoración Urbana (Presentación). UPC, Centro de Política de Suelo y Valoraciones, CPSV. Barcelona.

Maldonado, M.M. (agosto, 2000). "El Proceso de Construcción del Sistema Urbanístico en Colombia: entre Ordenamiento Territorial y Reforma Urbana". Trabajo presentado en el seminario El Marco Jurídico para la Construcción de Ciudad. Normativa Urbana y de la Vivienda, Montevideo. Resumen recuperado de: [http:// info.worldbank.org/etools/docs/library/135753/ M1-06-A-mariammaldonado.pdf]. 
Maldonado, M. M. (2008). "La Ley 388 de 1997 en Colombia: algunos puntos de tensión en el proceso de su implementación". Ace: architecture, city and environment $=$ arquitectura, ciudad y entorno, 3 (7), pp.43-66. Recuperado de: [http://upcommons.upc. edu/revistes/bitstream/2099/5634/1/3_MARIAMERCEDES3.pdf].

Ministerio del Medio Ambiente, Dirección General de Asentamientos Humanos y Población Subdirección de Medio Ambiente Urbano (1996) Lineamientos para una política ambiental urbana en Colombia (documento de trabajo). Recuperado de: [http://leensobell.files.wordpress. com/2008/09/politica_urbana.pdf].

Molina, L; Albarracín, C. (2008) "Los instrumentos de planeamiento urbanístico en la Ley 388 de 1997". Ace: Architecture, City and Environment = Arquitectura, Ciudad y Entorno, 3(7), pp. 67-78. Recuperado de: [http://www-cpsv.upc.es/ace/Arti cles_n7/articles_pdf/4_LUISM-CAROLINA.pdf].

Municipio de Sogamoso. Decreto No. 203 De 2002. Por el cual se reglamenta el conjunto de normas de urbanismo y construcción en suelo urbano y de expansión urbana. Recuperado de: [http:// sogamoso-boyaca.gov.co/apc-aa files/3035393138 3638653366373563613631/Decreto_Norma_ urbanismo_y_construccion.pdf].

Porter, M. (1990, marzo). “The competitive advantage of nations". Harvard Bussines Review, 68 (2). Recuperado de: [http://hbr.org/].

Presidencia de la República. Decreto 2181 de 2006. Por el cual se reglamentan parcialmente las disposiciones relativas a PPs contenidas en la Ley 388 de 1997 y se dictan otras disposiciones en materia urbanística. Diario Oficial 46320 de julio 05 de 2006. Recuperado de: [http://www.alcaldiabogota. gov.co/sisjur/normas/Norma1.jsp?i=20809].

Ramírez, J. F. (2011). "Historia crítica de la planeación urbana en Colombia. Una aproximación interpretativa desde los estudios sociales de la ciencia". Tesis de Maestría. Universidad Nacional de Colombia, Bogotá, Colombia. Recuperado de: [http://www.bdigital.unal.edu.co/5217/].

Rodríguez, D. (2007). Urbamedia: Base de datos de áreas centrales, casos de ciudades argentinas y latinoamericanas. Buenos Aires: Universidad de Buenos Aires.

Sabrino, J. (2005). "Competitividad territorial: ámbitos e indicadores de análisis”. Economía, Sociedad Y Territorio, (1), pp. 123-183. Recuperado de: [http://www.cmq.edu.mx/revistaest.html].

Tarchópulos, D. (2006) "Las huellas del Plan para Bogotá de Le Corbusier, Sert y Wiener". Scripta Nova [en línea], 10 (218). Recuperado de: [http:// www.ub.edu/geocrit/nova.htm].

Vejarano, M. C. (2012) Bogotá, localidad de Bosa. Unidades de Planeamiento Zonal de Mejoramiento Integral: urbanismo funcionalista? urbanismo inmobiliario? derecho a la ciudad? exclusión social? (UGI 2008, Proyecto No. 201010012032) Bogotá: Universidad Nacional de Colombia.

Yevenes, A. \& Andalaft, A. (2005) “Competitividad territorial y estrategia empresarial: el caso de la industria forestal exportadora de la región del BÍO BÍO”. Horizontes Empresariales, (5), pp. 39-51. Recuperado de: [http://www.horizontesempresariales.cl/]. 